\title{
EL DISEÑO DE NUEVOS AMBIENTES DE APRENDIZAJE Y EL DEBILITAMIENTO DE LAS FRONTERAS DE LA ESCUELA
}

\author{
Miller Antonio Pérez Lasprilla ${ }^{1}$
}

\begin{abstract}
Resumen: En el presente texto, a partir de la perspectiva genealógica y culturalista, se propone analizar, de una parte, por qué los nuevos ambientes de aprendizaje no han generado una apropiación masiva y significativa de los conocimientos útiles para el mercado y la eficiencia técnica (materias STEAM), y, de otra, cuáles son los aspectos que se deben considerar en el diseńo de un ambiente de aprendizaje para el aprendizaje de dichos conocimientos "útiles". Con este análisis se concluye que el debilitamiento de las fronteras de la escuela moderna debilita las posibilidades de aprender los conocimientos "útiles" para la productividad y la eficiencia técnica en nuevos ambientes de aprendizaje, al desfavorecer la experiencia que conlleva una relación particular consigo mismo y con los demás, que se encuentra en la base del aprendizaje formal, propio de la escuela. Además, se propone, que para el diseńo de nuevos ambientes de aprendizaje, es preciso considerar la codificación que enmarca la construcción estética sensorial, los elementos físicos, la temporalidad y el modo de interacción de los sujetos en un territorio delimitado, física e históricamente.
\end{abstract}

Palabras clave: Códigos, cultura, ejercicios, ambientes de aprendizaje, escuela.

\section{O DESENHO DE NOVOS AMBIENTES DE APRENDIZAGEM E O DEBILITAMENTO DAS FRONTEIRAS DA ESCOLA}

Resumo: No presente texto, a partir da perspectiva genealógica e cultural, propôe-se analisar, por um lado, por que os novos ambientes de aprendizagem não geraram uma apropriaçáo massiva e significativa de conhecimento útil para o mercado e eficiência técnica (sujeitos STEAM), e, por outro, quais os aspectos que devem ser considerados na criaçáo de um ambiente de aprendizagem para o aprendizado desse conhecimento "úteis". Com esta análise, conclui-se que o enfraquecimento das fronteiras da escola moderna enfraquece as possibilidades de aprendizagem do conhecimento "úteis" para produtividade e eficiência técnica em novos ambientes de aprendizagem, por desfavorecer a experiência que implica uma relação particular consigo mesma e com os outros, que está na base da aprendizagem formal, típica da escola. Além disso, propóe-se que, para a criação de novos ambientes

1 Profesor de la Maestría en Ciencias de la Educación de la Universidad de San Buenaventura, Colombia. Este trabajo hace parte de la reflexión inicial del autor para la definición de su tesis doctoral en el énfasis de Educación, cultura y desarrollo del Doctorado interinstitucional en Educación de la Universidad Pedagógica Nacional de Colombia. 
de aprendizagem, seja necessário considerar a codificação que enquadra a construção estética sensorial, os elementos físicos, a temporalidade e o modo de interação dos sujeitos em um território delimitado, física e historicamente.

Palavras-chave: Códigos, cultura, exercícios, ambientes de aprendizagem, escola.

\title{
THE DESIGN OF NEW LEARNING ENVIRONMENTS AND THE WEAKENING OF SCHOOL BOUNDARIES
}

\begin{abstract}
In the present text, from the genealogical and cultural perspective, it is proposed to analyze, on the one hand, why the new learning environments did not generate a massive and significant appropriation of useful knowledge for the market and technical efficiency (subjects STEAM) , and, on the other hand, what aspects should be considered in creating a learning environment for learning "useful" knowledge. With this analysis, it is concluded that the weakening of the frontiers of the modern school weakens the possibilities of learning "knowledge" for productivity and technical efficiency in new learning environments, because it disfavor the experience that implies a particular relation with itself and with the others, which is the basis of formal learning typical of the school. In addition, it is proposed that, in order to create new learning environments, it is necessary to consider the coding that frames the sensorial aesthetic construction, the physical elements, the temporality and the interaction mode of the subjects in a delimited territory, physically and historically .
\end{abstract}

Keywords: Codes, culture, exercises, learning environments, school.

\section{Introducción}

Durante la modernidad la escuela ha venido funcionando como un espacio físico donde hay aulas, generalmente, equipadas con un tablero, un escritorio y unas sillas alineadas para la configuración de un modo de interacción a partir de unos tiempos y unos ritmos que dan lugar a prácticas pedagógicas más o menos definidas. Sin embargo, debido a la educacionalización del mundo que consiste en asumir todos los problemas sociales y económicos como problemas educativos (Troller, 2014), la escuela ha sido objeto de continuas demandas de transformación de los espacios físicos y las dinámicas de interacción con dos propósitos fundamentales:

1. Incrementar el aprendizaje de los "conocimientos útiles" para solucionar problemas de la vida cotidiana, y

2. generar un mayor nivel de libertad y autonomía en los estudiantes en cambio del régimen disciplinario de la escuela moderna.

En relación con estos dos objetivos, desde hace años se asiste a un discurso en contra de la escuela moderna, soportado en gran medida por las nuevas formas de gobierno neoliberal, el cual demanda un espacio abierto y extendido para el pleno ejercicio de la libertad y la autonomía y el aprendizaje masivo de los "contenidos útiles" para la productividad y la eficiencia técnica.

De acuerdo con Drucker (1969), el primer objetivo deriva de considerar el aprendizaje como un capital basado en conocimientos útiles para el mercado; 
actualmente, estos conocimientos se refieren a las materias que identifican la sigla en inglés STEAM: Science, Technology, Engineering, Art y Mathematics, es decir, Ciencia, Tecnología, Ingeniería, Arte y Matemáticas. El segundo objetivo deriva de la dualidad entre individuo y sociedad que trajo consigo una forma liberal del proyecto moderno; este proyecto se basa en la posibilidad y la capacidad de tomar decisiones de forma libre y autónoma para distinguirse de los demás y contribuir al orden colectivo desde esa diferenciación.

Con este propósito, en los últimos años se ha venido promoviendo el diseño de nuevos ambientes de aprendizaje como una forma de reconfigurar el espacio y modos de interacción de la escuela moderna, con un especial uso de las Tecnologías de la Información y de la Comunicación (TIC) y la integración entre procesos de educación formal y no formal. En relación con el uso de las TIC, Vélez (2009) asegura que:

si bien es cierto que la noción de ambientes de aprendizaje también evoca aulas de clases dotados de mobiliario, equipamiento y determinado estilo de docencia, los nuevos ambientes de aprendizaje remiten más bien a formas diferentes de organizar los procesos de enseñanza y de aprendizaje que no tienen un correlato físico (p. 160) .

La integración entre la educación formal y no formal se ha planteado como una articulación de la comunidad en el marco de la escuela y bajo un concepto más amplio de currículo. Desde la perspectiva de Marcondes (2004) "la utilización del concepto de comunidad educativa posibilita una ampliación del concepto de educación, que no se restringe apenas a los procesos de enseñanza al interior de las unidades escolares formales" (p.40), dado que la comunidad constituye el espacio común para debatir los problemas sociales en la esfera pública. De esta forma, el diseño de nuevos ambientes de aprendizaje se plantea como una forma de debilitar las fronteras físicas, pero, también, del tiempo y los modos de interacción que configuró la escuela moderna con el fin de responder a los dos objetivos enunciados anteriormente.

Salinas (2008) señala un efecto de la integración entre el uso de las TIC y los espacios no formales el cual ayuda a entender el rumbo que ha tomado esta tendencia, en palabras del autor:

Las redes de comunicación introducen una configuración tecnológica que potencia este aprendizaje más flexible y, al mismo tiempo, la existencia de nuevos escenarios del aprendizaje. Del abanico de posibilidades que ofrecen podemos contemplar tres escenarios (Hiltz, 1992; Salinas, 1995): aprendizaje en casa, aprendizaje en el puesto de trabajo y aprendizaje en un centro de recursos de aprendizaje o centro de recursos multimedia. (p.5)

Estos escenarios a la vez se han formalizado en modalidades educativas asincrónicas y deslocalizadas como el e-learning y el blended learning con el fin de incrementar la cobertura y mejorar el aprendizaje permanente. Sin embargo, aunque se ha incrementado la experiencia de libertad en los nuevos ambientes de aprendizaje, salvo algunas excepciones importantes, hasta ahora la evidencia 
empírica no ha mostrado cambios o mejoras sustanciales en la apropiación masiva de los conocimientos útiles en los nuevos ambientes de aprendizaje. Recientes informes, por ejemplo, El Dividendo Digital (2015) realizado por los organismos multilaterales, como el Grupo Banco Mundial y que muestran resultados de estudios de impacto, concluyen que no hay cambios significativos en la apropiación de tales conocimientos útiles ${ }^{2}$ luego de integrar ciertos dispositivos electrónicos a la práctica educativa. Estos resultados se pueden contrastar con los datos de las pruebas comparadas de conocimiento, como las PISA ${ }^{3}$, en las cuales se observa, hasta hoy, la reproducción de datos históricos: los países del primer mundo obtienen los mejores puntajes y los países en vías de desarrollo siguen obteniendo bajos resultados a pesar del uso extendido de la tecnología y la integración de los espacios no formales en tareas de enseñanza.

En el mismo sentido, el informe de la Comisión Europea (2008) resalta los deficientes resultados que se han obtenido con el diseño de los nuevos ambientes de aprendizaje:

El impacto de las TIC en la educación y en la formación todavía no ha sido tan significativo como se esperaba a pesar del amplio apoyo político y social que han tenido [...] La inserción de las TIC en la educación y en los sistemas de formación requiere nuevos cambios a través de ámbitos como el tecnológico, organizativo, enseñanza y aprendizaje en las aulas, en los lugares de trabajo y en los entornos de aprendizaje informal. A pesar de que las TIC tienen el potencial para desarrollar un proceso continuo de aprendizaje que apoyaría el aprendizaje permanente, abarcando el aprendizaje formal, informal y en el lugar de trabajo, esto aún no ha sido alcanzado. (p. 4)

al decir que,

en realidad, el problema no es que las promesas no se hayan cumplido, sino
que la manera en que se ha llevado a cabo la incorporación de la tecnología no
ha sido las más adecuados. Los resultados y conclusiones de la investigación
lo evidencian con datos muy claros: incorporar TIC no tiene una relación
directa con la innovación educativa ni con la obtención de mejores resultados
académicos. (p. 157)

Por lo tanto, es necesario preguntarse, ¿por qué los nuevos ambientes de aprendizaje no han generado una apropiación masiva y significativa de los conocimientos útiles para el mercado y la eficiencia técnica (materias STEAM) que se espera? Y en esa misma línea de posibilidad, ¿cuáles son entonces los aspectos que se deben considerar en el diseño de un ambiente de aprendizaje para el aprendizaje de los conocimientos útiles?

2 El proyecto One Laptop per-Child en el Perú proporcionó cientos de miles de equipos de computación de bajo costo a escuelas rurales. Pero las primeras investigaciones no encontraron evidencia de un mayor aprendizaje en matemáticas o en el lenguaje. (Cristia y otros 2012, citado por el Grupo Banco Mundial, 2016, p. 146)

3 Ver informe de resultados: http://www.oecd.org/pisa/pisa-2015-results-in-focus-ESP.pdf 
Para responder a estas preguntas y considerar las posibilidades de diseño que ofrece el concepto de ambiente de aprendizaje, en este texto se propone un análisis a partir de la perspectiva genealógica y culturalista que identifica los aspectos de la emergencia y la configuración del concepto de ambiente de aprendizaje en el marco de la construcción histórica de la escuela moderna.

\section{Perspectivas teóricas para el análisis}

El diseño de ambientes de aprendizaje se suele analizar por lo menos en dos sentidos, uno, que permite reconocer los elementos que configuran y dan forma a un determinado ambiente, y otro que permite al maestro disponer el ambiente para lograr un efecto significativo en el aprendizaje de sus estudiantes. En el primer caso, se habla de ambientes históricamente constituidos y, en el segundo, de ambientes diseñados por el maestro para desarrollar su tarea educativa. Con respecto a esta segunda opción, (Moreno, 2008, citado por García-chato, 2014) afirma que:

un ambiente de aprendizaje se diseña para desarrollar procesos intencionados de aprendizaje no accidentales, procurados para crear condiciones pedagógicas y contextuales favorables al proceso de aprendizaje del niño, con base en un currículo configurado por el contenido conceptual, actitudinal, procedimental y las aportaciones culturales que ofrece la escuela. (p. 71)

Frente al primer aspecto, Marín-Díaz y Noguera (2017) plantean una interpretación de las tesis de Simons y Masschelein (2014), en la que el surgimiento de la escuela moderna sirve para el análisis de los ambientes históricamente constituidos. Para ello, primero, muestran la emergencia de la escuela como resultado de una forma nueva y particular de pensar la formación de los seres humanos y, segundo, se ocupan del problema del aparente debilitamiento de las fronteras que se establecieron para la escuela en el proyecto moderno, para mostrar que precisamente estos límites, que son espaciales, de contenido y de régimen disciplinar, resultan necesarios para que los sujetos se puedan autorregular a fin de construir aquello que Simons y Masschelein (2014) denominan lo público y el bien común.

En el segundo caso, con respecto al diseño que realiza un maestro sobre el ambiente de aprendizaje, es posible acudir a conceptos y categorías culturalistas que permiten analizar e identificar la codificación de los ambientes de aprendizaje para prever la posibilidad de diseño. En esta línea, las posibilidades de diseñar un ambiente de aprendizaje vendrán de la mano de las opciones para variar la codificación y los modos de interacción que permitan obtener los resultados esperados.

\subsection{Emergencia del concepto de ambiente de aprendizaje}

En el marco de configuración de la escuela moderna puede observarse una relación entre las ideas de Comenio (1994) y las ideas de Rousseau (1984) sobre la educación de los niños que permite identificar la emergencia del concepto de 
ambiente de aprendizaje. Pues, por un lado, se observa la necesidad de un régimen disciplinar orientado por un maestro en un espacio destinado para este fin y, por otro lado, se comprende el proceso como una acción indirecta que ocurre en el medio natural, lejos del espacio convencional de lo que se conoce ahora como un edificio escolar. Mientras el proyecto de Comenio destaca la importancia del entendimiento de las cosas; en cambio Rousseau, en su libro Emilio, no da importancia a los contenidos y se concentra en el desarrollo de unas supuestas disposiciones internas dictadas por la naturaleza, para lo cual se requiere una acción indirecta en un medio natural.

\section{Para Rousseau (1984):}

La educación es efecto de la naturaleza, de los hombres o de las cosas. La de la naturaleza es el desarrollo interno de nuestras facultades y nuestros órganos; la educación de los hombres es el uso que nos enseñan éstos a hacer de este desarrollo; y lo que nuestra experiencia propia nos da a conocer acerca de los objetos cuya impresión recibimos, es la educación de las cosas. (p. 10)

Bajo la perspectiva de este texto, entre estas dos formas de entender la educación, puede observarse la emergencia del concepto de ambiente de aprendizaje que parece orientar las acciones que buscan masificar el aprendizaje de los "conocimientos útiles". Ya que por un lado se retoma el principio de acción indirecta de Rousseau, y con ello la influencia del entorno en la apropiación y recreación de significados, y, por el otro, se acentúa el entendimiento de las cosas que promovió Comenio en su Didáctica Magna (enseñar todo a todos). De manera que diseñar un ambiente de aprendizaje podría entenderse como una forma de usar todo aquello que rodea el sujeto para lograr un efecto indirecto en su aprendizaje formal.

No obstante, si se entiende así, este no sería más que un arreglo técnico que pondría en contradicción los planteamientos originales de los dos autores, ya que Comenio plantea la necesidad de un régimen disciplinario que implica un aislamiento en un ambiente hecho por el hombre para superar la animalidad y alcanzar la humanidad; y por su parte, Rousseau no pretendía enseñar un contenido sino favorecer el encauzamiento natural de las disposiciones internas para el ejercicio de la libertad y la autonomía en el espacio regular de interacción.

Ahora bien, si se sitúa esta discusión en el enfoque neoliberal de la educación, en el cual no se aprenden contenidos, sino que se aprende a aprender para poder adaptarse con facilidad a los cambios del mercado, la contradicción es mucho mayor porque desde esta perspectiva no se aprende ningún contenido, como señala Comenio, sino que se aprende o se pretende aprender una forma de asimilar y desaprender contenidos según la exigencia del mercado. Frente a este escenario, ¿es posible que la perspectiva neoliberal de la educación esté persiguiendo el proyecto de Comenio con el método de Rousseau a la hora de plantear nuevos ambientes de aprendizaje? Si es así, podría estar cayendo en una profunda contradicción.

Rousseau (1984) apelaba a la naturaleza para educar a Emilio porque consideraba este escenario como la posibilidad de revelar lo que ya poseía, es decir, 
la disposición natural a realizarse. Sin embargo, no se trataba de una exploración espontánea sino de una exposición arreglada por él para obtener determinados efectos indirectos. Desde su perspectiva, para no interferir en la educación de Emilio, era preciso no intervenir de forma directa sino a través de los elementos de su entorno cotidiano, con lo cual revela dos aspectos fundamentales para lo que hoy se conoce como diseño de ambientes de aprendizaje: a) el efecto indirecto del medio natural y social y b) el arreglo que hace el maestro para generar determinados efectos en su estudiante.

Con respecto al primer punto, el efecto indirecto es uno de los aportes más significativos de Rousseau a la educación y la pedagogía de su tiempo. Para el autor, es preciso no enseñar para que el otro pueda aprender; asimismo, la intención explícita del maestro, para que el estudiante aprenda, es antinatural y perjudicial para su educación; por lo tanto, se debe encontrar la forma de provocar situaciones en medio de la interacción cotidiana que involucren al estudiante en una búsqueda de respuestas a preguntas subyacentes.

Sin embargo, si se extrapolara el concepto de diseño de ambiente de aprendizaje de la obra de Comenio, no coincidiría con la propuesta de Rousseau, porque, en primer lugar, Rousseau no se proponía enseñar nada a su estudiante, ya que el propósito del diseño de la situación en un medio natural era provocar un desenvolvimiento de la capacidad de revelar su naturaleza y no que aprendería determinados temas o conceptos. En cambio, el método y los ejercicios del llamado "taller de humanidad" propuesto por Comenio, se presentan como formas de acceder a todos los conocimientos que se encuentran o debería encontrarse en posesión o dominio de un maestro erudito que conoce el fundamento de las cosas. De manera que las técnicas, ejercicios, tiempos y espacios destinados a desarrollar tareas como tomar la lección, reelaborar el contenido y participar en una discusión, por nombrar algunas técnicas y procesos, constituyen un planteamiento metodológico para la apropiación de un conocimiento de dominio especializado que al parecer debe enseñarse en un espacio aislado y bajo reglas y normas específicas; es decir, en la escuela y no en cualquier medio de interacción cotidiana como sugiere Rousseau.

Pero ¿esto quiere decir que los autores presentan dos formas diferentes de aprender lo mismo? O ¿Podría decirse que se aprende algo distinto con cada perspectiva? Una breve revisión del concepto de educación propuesto por cada autor podría ofrecer unas pistas para responder a estas preguntas.

En la obra de Rousseau (1984), el verdadero fin de la educación es aprender a ser libre y autónomo, como lo afirma el autor:

El único que hace su voluntad es el que para hacerla no necesita de auxilio ajeno; de donde se infiere que el más apreciable de los bienes no es la autoridad, sino la libertad. El hombre verdaderamente libre sólo quiere, lo que puede y hace lo que le conviene. Esta es mi máxima fundamental; trato de aplicarla a la infancia y veremos derivarse de ella todas las reglas de la educación. (p.78) 
Mientras que en la obra de Comenio (1994) el fin de la educación es revertir la animalidad con la cual llegamos al mundo para ser libres: "quede, pues sentado, que a todos los que nacieron hombres les es precisa la enseñanza, porque es necesario que sean hombres, no bestias feroces, no brutos, no troncos inertes" (p. 23).

En ambos casos el fin de la educación es lograr la libertad y la autonomía, pero con un planteamiento distinto de la interacción entre el maestro y el estudiante que conlleva una relación diferenciada con los objetos de conocimiento. Rousseau planteaba una relación individual, un maestro para un estudiante en el espacio cotidiano de interacción sin un plan de estudios determinado, incluso, lejos de la ciudad con un alcance limitado en cobertura. Podría decirse, que se trata de una educación de élite basada en la antigua figura del preceptor o en la relación que puede establecer hoy un padre o una madre con su hijo. Por el contrario, Comenio planteó el "taller de humanidad" como una forma de enseñar todo a todos, lo cual quiere decir que no deja a nadie por fuera e incluye no sólo los saberes de la vida cotidiana, sino también aquellos saberes que derivan de la erudición.

Para este propósito, a Comenio no le resulta suficiente el espacio, el tiempo y el modo de relación de la vida cotidiana, es así que plantea la necesidad de un lugar regido por unas técnicas disciplinarias que, de acuerdo con Noguera y Marín-Díaz (2017), constituyen "las ascesis, formas de ejercitación que llevan al moldeamiento de la vida a través de actividades repetidas y reguladas, [que] se constituyeron en el eje que caracteriza la vida escolar" (p. 612).

Al respecto, Noguera y Marín-Díaz (2017) afirman que:

Los modos que tales ascesis adoptaron, y la manera cómo funcionaron y funcionan en las sociedades modernas, estuvieron vinculados y se hicieron posibles con la aparición de uno de los principales artefactos utilizados para la producción de eso que reconocemos como propiamente humano: la escuela moderna (p. 612)

De manera que la escuela de Comenio parece plantearse como una forma de aprender algo diferente de lo que se aprende en la vida cotidiana con Rousseau. Las dos perspectivas aspiran a generar un mayor nivel de autonomía y libertad en los estudiantes, pero a través de técnicas, ejercicios, espacios y formas de interacción diferenciados. A primera vista, la diferencia podría estar en la intención explícita e implícita del maestro que se juega en la interacción. No obstante, en la perspectiva de Comenio la intención del maestro se hace evidente en las lecciones y la disposición del espacio y el tiempo de la escuela, pero, en Rousseau esta intención aparece velada en la interacción espontánea que percibe el estudiante. Después de revisar estas dos propuestas, se concluye que la diferencia podría estar en la percepción, aparente, de ser libre y autónomo de la experiencia Rousseauniana versus la experiencia de sentirse controlado y dirigido en la escuela de Comenio.

Para el propósito de este texto, esta diferencia aparece en el centro mismo del debilitamiento de la escuela moderna con el diseño de nuevos ambientes de aprendizaje, especialmente, con el uso de las TIC y la integración de los espacios formales y no formales, porque la flexibilización del espacio, el tiempo y el modo 
de interacción de la escuela no sería más que la intención explícita de generar una mayor experiencia de libertad y autonomía de los estudiantes al modo de Rousseau. Sin embargo, queda aún por resolver si hay alguna relación entre la forma histórica la escuela y el aprendizaje de los "conocimientos útiles" para la productividad y la eficiencia técnica, para lo cual es preciso preguntarse: ¿es posible que la diferencia entre el planteamiento educativo de Rousseau y Comenio también resida en los objetos de conocimiento que se delimitan, apropian y construyen con base en técnicas, ejercicios y modos de interacción diferenciados?

Para responder a esta pregunta, resulta necesario acudir a la configuración histórica de la escuela moderna en busca de las particularidades que permitan llegar a una respuesta.

\subsection{Configuración histórica del ambiente escolar}

Foucault (1978) expuso que la escuela es un invento moderno que adoptó su forma de las escuelas monásticas, entre otras instituciones. De esta forma, la institución escolar heredó una arquitectura y, sobre todo, unos ejercicios que con mayor o menor variación se han mantenido como aspectos constitutivos del ambiente escolar. Para el propósito de este texto, estos elementos pueden organizarse alrededor del concepto de ambiente escolar, entendido como la configuración histórica de una disposición de elementos en un espacio físico, una temporalidad y una forma de relacionarse que provoca cierta reacción y adaptación de los seres humanos en relación con una construcción cultural bajo un propósito de conducción o relación consigo mismo y los demás.

De acuerdo con Daepe \& Simmons (2008) la escuela ha tomado forma frente a la "vida" en torno a los ejes del tiempo, el espacio y las formas de interacción que han establecido ciertas pautas de comportamiento. A esta perspectiva habría que añadir que el saber que circula en la escuela obedece a una forma de erudición que le otorga su especificidad.

a) En relación con el tiempo se ha producido una configuración de largo y corto plazo en el desarrollo del currículo referido a los objetivos semanales, bimestrales y anuales, que se alternan con periodos de vacaciones $\mathrm{o}$ recesos. De igual forma, se cuenta la distribución de las horas de clase, los recreos y la hora de almuerzo, entre otros aspectos.

b) En relación con el espacio se reconoce una disposición arquitectónica de espacios como los salones de clase, el patio de recreo, el gimnasio, el auditorio, entre otros, que obre todo cumplen una función comunicativa en el modo de interacción pedagógica y didáctica dispuesto. Así, por ejemplo, puede hablarse de los "espacios mentales" creados para comunicar cuáles son los límites y marcos normativos para la acción, tales como las márgenes en color rojo de los cuadernos para indicar hasta donde se debe escribir, o las líneas hechas en el patio de recreo para establecer de qué forma debe hacerse la fila. También, pueden destacarse aspectos como la estructura de claustro de la escuela, que representa un encerramiento, 
un espacio protegido, un lugar aislado del resto de la vida para realizar ejercicios; es un lugar en el que se entregan los niños al cuidado de unos adultos, o un espacio de intercambio entre el resto de la sociedad y la institución escolar.

c) El nivel de la interacción y la comunicación configura un tipo de relación pedagógica y didáctica en dos niveles, referidos al intercambio verbal y el no verbal. Con respecto al primero, se puede reconocer la existencia de unas formas de subjetivación (tono, sentido de lo dicho...) ligadas en algún momento a la teología, en cuanto forma de salvar y ayudar al niño a encontrar sentido a la existencia, o a la perspectiva médica que intentó darle una base científica a la pedagogía para replantear los marcos normativos del ambiente escolar, en favor de una autorregulación y auto direccionamiento. En consecuencia, esta primera perspectiva configuró la existencia de un maestro pastor, en cambio, la segunda perspectiva proyectó la figura de un maestro animador (Depaepe y Simmons, 2014). De cualquier manera, de estas dos formas de subjetivación se desprenden gestos que configuran un ambiente escolar, tales como levantar el dedo en señal de advertencia, dar una palmada en la espalda en señal de aprobación, lanzar una mirada fija como sanción moral o sonreír como forma de aprobación.

Por ende, la articulación de estos tres ejes ha determinado unas características que definen en gran medida la "experiencia escolar". Según Marín-Díaz y Noguera (2017) estos ejes definen lo escolar y pueden clasificarse de la siguiente manera:

a) una forma de relación con el conocimiento heredado por las generaciones anteriores, b) una práctica vinculada con la vida reglar, con las normas, la disciplina y la ejercitación, y c) la producción de unos modos específicos de subjetivación, una apertura al mundo. (Marín-Díaz y Noguera, 2017, p. 617)

Para el primer aspecto, en el ambiente escolar se aprende, sobre todo y de manera diferencial, el conocimiento erudito referido por Comenio. Si bien, en el marco amplio de la cultura el sujeto se apropia de los saberes acumulados por la humanidad, es en el ambiente escolar donde se apropia, de manera diferenciada, aquello que no puede tomarse en la interacción cotidiana. En este punto también se incluyen los saberes para la conducción de sí mismo y la autorregulación, estos se presentan en forma de valores y preceptos de comportamiento, así como los aspectos relacionados con los saberes para la relación consigo mismo y los demás. Sin embargo, el conocimiento erudito sería lo verdaderamente único y particular del ambiente escolar, porque mientras otras instituciones sociales diferentes a la escuela, se abrogan la tarea de "educar", ninguna puede reemplazar por completo esta tarea específica.

El segundo aspecto relacionado con la disciplina se entiende con Foucault como una confluencia de fuerzas para la conducción de la vida que, en últimas, produce formas de ser y estar con los otros. Según Noguera y Marín-Díaz (2017): 
En esta perspectiva, la experiencia escolar supone una aproximación con la norma, que permita regular, orientar y gobernar las fuerzas y orientarlas en acciones. Ese es el valor de la norma y la disciplina. Ellas son condición para la novedad, para el pensamiento, para lo otro. Así, en contra de las profecías de una sociedad libertaria fundadas en una mirada ingenua, paranoica y hedonista contra la norma y la disciplina, el dispositivo de formación escolar permite reconocer la potencia de las antropogénicas disciplinares, su capacidad productora de novedad y transformación. (p.619)

El tercer aspecto, se relaciona con la "la forma-de-vida, la aceptación de la conducción de sí mismo y de los otros como modo de vida, como forma de ser y estar en el mundo y con los otros" (Marín-Díaz y Noguera, 2017, p. 619), referida a un tipo de interacción que conlleva un modo especial de autorregulación y relación con los demás.

En síntesis, el ambiente escolar parece producir una forma particular de autorregularse y dirigirse con lo cual se puede establecer una especial relación con las formas del conocimiento erudito que permite no solo apropiar de los significados, sino también hallar la novedad en ellos. De esta forma, las técnicas, los ejercicios y los modos de relacionamiento en el espacio físico de la escuela, parecen producir una experiencia de ser y estar con los otros lo que constituye una base para el aprendizaje permanente. En este sentido, es muy esclarecedor que en los nuevos ambientes de aprendizaje que configura el uso de las TIC y la integración de los espacios no formales, se exija a los estudiantes habilidades para el aprendizaje autónomo, entendido como: "[...] la facultad de dirigir el propio proceso para entender y comprender la realidad" (Sierra Pérez, 2005, p.3). Es decir, para poder aprender significativamente en los nuevos ambientes de aprendizaje, el estudiante se debe autorregular y conducirse por su cuenta.

Pero ¿esto quiere decir que la "experiencia escolar" es una condición para poder aprender los "conocimientos útiles" de manera autónoma en los nuevos ambientes de aprendizaje? Si es así, se estaría frente a una gran paradoja porque significa que el debilitamiento de las fronteras de la escuela moderna sería el principal obstáculo para poder aprender masivamente los contenidos de las materias STEAM que promueve la perspectiva neoliberal de la educación.

Por ejemplo, si se piensa en un académico, se tiene el estereotipo de una persona que pasa muchas horas sentada estudiando, escribiendo textos y discutiendo conceptos y categorías con otros académicos, entonces, ¿qué tipo de autorregulación se requiere para poder estudiar de esa forma? Pues bien, parece ser que la experiencia escolar no solo está en la base de la participación en lo público y común, como menciona Simons y Masschelein (2014), sino que también podría estar vinculado con el tipo de ejercitación y autorregulación necesaria para poder aprender ciertos contenidos de la tradición académica. Es así que debilitar la forma de la escuela moderna, también estaría debilitando la posibilidad de aprender los "conocimientos útiles" en los nuevos ambientes de aprendizaje porque desde esta perspectiva, la capacidad de aprendizaje autónomo no sería otra cosa que la experiencia escolar actuando como forma de vida. 
Hasta este punto se ha intentado responder a la primera pregunta que deriva de la problematización del texto, ésta es, ¿por qué los nuevos ambientes de aprendizaje no han generado una apropiación masiva y significativa de los "conocimientos útiles" para el mercado y la eficiencia técnica (materias STEAM) que se espera? A partir de las conclusiones preliminares, se puede sostener que el debilitamiento de las fronteras de la escuela moderna también debilita las posibilidades de aprender los conocimientos "útiles" para la productividad y la eficiencia técnica en nuevos ambientes de aprendizaje. Este debilitamiento desfavorece la experiencia que conlleva una relación particular consigo mismo y los demás, condición que se encuentra en la base del aprendizaje formal propio de la escuela.

Aunque esto no significa que el uso de las TIC en tareas de enseñanza y aprendizaje, y la integración de los ambientes formales y no formales, dejen de representar una alternativa importante para dinamizar procesos educativos; por el contrario, se sugiere que esa integración se debe producir a partir del reconocimiento de los aspectos que diferencian y constituyen el ambiente escolar para no perder de vista su especificidad. De esta consideración deriva la pregunta por ¿cuáles son los aspectos que se deben considerar en el diseño de un ambiente de aprendizaje para el aprendizaje de los "conocimientos útiles"? Esta pregunta se formuló en la problematización.

Con el fin de proponer una respuesta que incluya el ambiente escolar moderno, a continuación, se apela al concepto mismo de ambiente y sus características en relación con la experiencia estética sensorial, la cultura, el medio natural y las relaciones con el ambiente escolar histórico, a fin de proponer una clasificación culturalista de los ambientes de aprendizaje.

\section{Construcción culturalista del concepto de ambiente de aprendizaje}

Para Duarte (2003), el concepto de ambiente de aprendizaje es producto de una relación interdisciplinaria que vincula la ecología, la perspectiva ambiental de la educación y la psicología, en el marco del enfoque sistémico de la teoría del currículo, entre otras disciplinas. Aparentemente, el término de ambiente fue introducido por geógrafos en 1924 para describir aquello que resulta de la relación entre los seres humanos y el entorno natural que los rodea (Raichvarg, 1994), desde entonces se ha venido usando para comprender y plantear escenarios que trascienden el aprendizaje ligado a la forma histórica de la escuela moderna ${ }^{4}$. Con este concepto, se han querido incluir espacios sociales como el mercado, el museo, la calle, la iglesia, entre otros, en las actividades de enseñanza, dado que, como menciona García Chato (2014), "los elementos naturales, sociales, culturales,

4 En la primera parte del texto se plantea que, en la tradición pedagógica moderna, el concepto de Ambiente de aprendizaje formal es una conjunción entre aspectos de las tesis de Rousseau y Comenio. Sin embargo, ni Rousseau ni Comenio hicieron alguna referencia a los ambientes de aprendizaje - aunque sí establecieron principios para darle forma a la idea en la tradición pedagógica- por esa razón, en esta parte del texto se identifica la primera vez que aparentemente se usó el término para identificar este concepto. 
psicológicos y pedagógicos conforman el ambiente del hombre en y desde los cuales vive situaciones y procesos que dan lugar a la asimilación, transformación, recreación y socialización de la cultura" (p. 66). Como se mencionó anteriormente, esta integración se ha venido dinamizando con un especial uso de las TIC y la integración entre educación formal y no formal.

En este sentido, los ambientes de aprendizaje suelen entenderse como todo aquello que pueden percibir los sujetos, es decir, los olores, las formas, los colores, entre otros aspectos de la apropiación del mundo, en el marco social, físico e histórico de los sujetos en interacción. En suma, puede entenderse como el resultado de las percepciones de los diferentes miembros de un grupo o colectivo que generan actitudes, creencias y posicionamientos frente a lo que ocurre a su alrededor. De este modo, se acentúa la configuración del ambiente de aprendizaje con base en el dispositivo sensorial del organismo humano, pero, sobre todo, en el devenir histórico y la construcción cultural que separa y une al hombre con la naturaleza; porque, aunque el término "ambiente" suele usarse como sinónimo de medio y "naturaleza", no todos los expertos se ponen de acuerdo. Por ejemplo, Simons (1997) sostiene que la naturaleza se refiere a "todos los elementos y procesos de la tierra fuera de la especie humana" (p. 11) y que el ambiente corresponde al entorno alterado o intervenido por el hombre, mientras que otros autores como Reboratti (2000) sitúan esta diferencia como un simple aspecto general y particular de la misma naturaleza, sin que medie la cultura humana.

Con lo anterior se configuran dos escenarios para la discusión que se propone en este artículo: a) el ambiente humano como construcción estética sensorial b) y la diferencia entre naturaleza, ambiente y cultura.

\subsection{El ambiente humano como construcción sensorial y estética}

Como se mencionó anteriormente, para la ecología los ambientes se constituyen esencialmente por la relación que establecen los seres vivos entre ellos y con los demás elementos que conforman la biosfera y la ecosfera en un territorio específico, pero en el caso de los seres humanos aparece la cultura como mediación entre la "naturaleza" y el hombre, por lo cual se obtienen dos características esenciales de los "ambientes humanos": primero, los espacios físicos concretos, y segundo, la relación que establecen los sujetos con otros sujetos y los elementos del medio a través del dispositivo sensorial que comparte con los demás seres vivos.

Leroi-Gourham (1971) afirma que todos los seres vivos comparten por lo menos tres planos sensoriales referidos al comportamiento nutritivo, afectivo y el de integración espacial. "Estos tres planos de la estética fisiológica ponen en juego, en relaciones variables, los diferentes instrumentos del dispositivo sensorial: sensibilidad visceral, sensibilidad muscular, gustación, olfato, tacto, audición, equilibrio y visión" (p. 276).

En los seres humanos, la sensibilidad visceral y muscular marca un ritmo en la integración del tiempo y el espacio que conserva una relación con el proceso de valoración estética que une a cada sujeto con un determinado grupo étnico, 
es decir, "los ritmos son creadores del espacio y del tiempo, por lo menos para el sujeto; espacio y tiempo no existen como vividos sino en la medida en la cual son materializados en un envoltorio rítmico" (Leroi-Gourham, 1971, p. 301). Cuando habla de ritmos, este autor se refiere al movimiento de nuestra organicidad en procesos básicos como la nutrición, la afectividad y la integración del espacio, los cuales implican los momentos de alimentación, de relación con los otros y la misma actividad de percepción. Desde esta perspectiva, la marcha marca el ritmo de apropiación espacio temporal, de la cual derivan los conceptos como el kilómetro y hora, mientras la animación rítmica del brazo abre la posibilidad de crear las formas expresivas típicamente humanas. Así, compases, golpes espaciados y formas continuas marcan la diferencia entre el espacio natural y el espacio humanizado.

El olfato resulta ser un proceso de recepción que no cuenta con un mecanismo de expresión, y por eso, aunque se pueda simbolizar a través de la reflexión, su sentido resulta intransmisible. Sin embargo, aunque no hay recuerdos olfativos, el estímulo olfativo si revive imágenes y sonidos olvidados porque los ambientes tienen un olor particular. Por ejemplo, en lugares como la cocina se producen y mezclan diferentes olores que reviven viejos recuerdos que participan de la experiencia estética. Lo mismo ocurre con el ambiente escolar, por ejemplo, en este espacio se percibe el olor a temperas, hojas de papel, marcadores, entre otros, que actúan como elementos integradores del espacio, el tiempo y los recuerdos de todos los que participan de las actividades.

En tanto, el tacto participa de la integración espacio temporal y en la apreciación estética de las superficies ligado al sentimiento de caricia (ligera, suave, cadenciosa que transmite comodidad) y aunque no resulta definitivo para la integración del universo simbólico, sí participa en la apreciación estética de la cercanía con el otro, en las caricias o el simple contacto físico.

Mientras el gusto participa en el reconocimiento espacio temporal a través de la reacción de las papilas gustativas, éstas alertan del peligro de las sustancias peligrosas y permiten reconocer los saberes elementales, por ejemplo, lo dulce, lo salado o lo amargo.

Por último, la audición y la visión son registros sensoriales que cuentan con un sistema de recepción y emisión con base en la memoria y la relación dual entre la cara y la mano que permite la figuración. En términos de la trasmisión de la información, es preciso decir que tanto las imágenes como los sonidos pueden ser registrados y conservados en sistemas de retención terciaria, estos van desde cualquier tipo de superficie hasta los sistemas electrónicos de almacenamiento, mientras que las experiencias olfativas, gustativas y táctiles, siempre dependen de la percepción directa de los sujetos.

De esta forma, se observa que la construcción de los ambientes humanos se encuentra atada a las posibilidades y características del sistema perceptivo en cuanto a la recepción, figuración y conservación del universo simbólico. Por esa razón, tanto el reconocimiento de los ambientes históricamente constituidos, como las posibilidades de diseño de los ambientes de aprendizaje, dependen, en gran 
medida, de los elementos del sistema perceptivo que condicionan la integración de la experiencia del tiempo, el ritmo y el espacio en los seres humanos.

\subsection{La diferencia entre naturaleza y ambiente}

Para continuar esta discusión es preciso preguntarse ¿el hombre procede de la naturaleza o es un fenómeno disruptivo que termina alterándola?

Desde una perspectiva evolutiva es evidente que el hombre es una especie que ha tenido una transformación adaptativa que le confiere unas características particulares, las cuales derivan, precisamente, de condiciones ambientales. Lo que puede llamarse el camino evolutivo es una confluencia entre los elementos de la naturaleza: el clima, la presencia de otras especies, la comida, entre otras; y las características del organismo humano que se han transformado en la interacción con otros seres vivos y los elementos físicos del entorno. En este punto, puede decirse que el aprendizaje resulta ser un proceso adaptativo a condiciones impuestas por el medio que entran en relación con características del organismo afectado.

Sin embargo, el hombre no es solo un organismo adaptativo, a él lo distingue de los demás seres vivos la posibilidad de trascender debido a la facultad del lenguaje. $\mathrm{Al}$ respecto, Virno (2005) sostiene que, en el desarrollo evolutivo, el hombre detuvo la especiación adaptativa y desarrolló la posibilidad de exteriorizar el pensamiento a través de símbolos, con lo cual pudo construir significados, herramientas..., en fin, la cultura; pero no por eso el proceso de transformación ha dejado de ser "natural", simplemente siguió una ruta evolutiva distinta: una ruta cultural.

Para sortear la diferencia que existe entre naturaleza y ambiente puede decirse con Reboratti (2000) que la

naturaleza sería el conjunto de los elementos y relaciones terrestres en general,
sin una limitación territorial ni temática específicas, mientras que ambiente se
referiría al conjunto de elementos y relaciones biológicos y no biológicos que
caracterizan una porción de la tierra o que rodean y permiten la existencia de
un elemento (p.12).

Esta diferencia se queda en el nivel de la ecología, pero debe recordarse que el concepto de ambiente se usa en esta disciplina científica. No obstante, lo que puede agregarse en este punto, es que en realidad se trata de una diferencia entre la naturaleza y los ambientes humanos, es decir, los ambientes culturales.

\subsection{La diferencia entre naturaleza y cultura}

Como se dijo anteriormente, la facultad de lenguaje del ser humano se inscribe como un factor diferencial frente al resto de los seres vivos porque constituye la posibilidad de dar significado a la experiencia, producir herramientas, crear instituciones sociales y sistemas de valores que conllevan la transformación de la naturaleza a la medida de sus necesidades y aspiraciones. En este caso, hablamos de producción cultural como un conjunto de significados y técnicas que modifican la relación del hombre con la naturaleza en una vía diferente a la simple 
adaptación de los organismos y las especies en un ecosistema. De esta forma, la cultura se constituye como el medio de relación entre el hombre y la naturaleza, en contraposición a la adaptación de su organismo a los estímulos del medio.

En este sentido, Lévi-Strauss (1993) señala que la capacidad del hombre para extraer conclusiones generales a partir de la experiencia y establecer reglas particulares que definen las formas de vida que pueden ser transmitidas, asimiladas y reproducidas por otros miembros de la especie, es lo que realmente separa los seres humanos de los demás seres vivos, "En todas partes donde se presente la regla sabemos con certeza que estamos en el estadio de la cultura" (p.41).

Es así como para los seres humanos el medio ambiente no existe más allá de la cultura como un elemento inalterado, porque es por definición una red de significados que el hombre ha construido en interacción (Geertz, 1973). Por lo tanto, no existe nada por fuera de la cultura, porque todo lo que puede ser nombrado pasa por el lenguaje y pertenece a un orden arbitrario de significación, así corresponda a una manifestación de la Naturaleza. De esta forma, si se adopta la definición del lenguaje como una capacidad innata de los seres humanos para exteriorizar el significado a través de símbolos (Chomsky, 1998), tenemos como principio un sistema de expresión y contenido que permite establecer una convención o relación entre estos dos funtivos. Inicialmente, Saussure (1982) lo estableció como una diferencia y relación básica entre significante y significado, y luego Hjelmslev (1974) lo planteo como una diferencia entre expresión y contenido.

\subsubsection{Formas de apropiación y recreación de la cultura}

En el marco de estos presupuestos, Umberto Eco (2000) retomó el concepto de código como regla que permite establecer y reproducir la convención entre la expresión (materialidad y forma) y el contenido (forma y sustancia), para establecer las formas de la cultura.

En el siguiente cuadro se presenta la estructura fundamental de este planteamiento:

\begin{tabular}{|c|c|c|c|}
\hline \multicolumn{2}{|c|}{ Expresión } & \multicolumn{2}{|c|}{ Contenido } \\
\hline $\begin{array}{l}\text { Sustancia o } \\
\text { materialidad }\end{array}$ & Forma de la expresión & Forma del contenido & $\begin{array}{l}\text { Sustancia del } \\
\text { contenido }\end{array}$ \\
\hline $\begin{array}{l}\text { Soporte material } \\
\text { de la expresión: } \\
\text { papel, madera, } \\
\text { piedra, pantallas } \\
\text { electrónicas ... } \\
\text { Soportes e } \\
\text { instrumentos. }\end{array}$ & $\begin{array}{l}\text { Forma que toma la } \\
\text { expresión: } \\
\text { Icónica / Figurativa } \\
\text { Alfabética / abstracta }\end{array}$ & $\begin{array}{l}\text { Forma en que se organizan } \\
\text { los significados: } \\
\text { Figurativa/ Narrativa } \\
\text { Abstracta / Argumentativa }\end{array}$ & $\begin{array}{l}\text { Lo que representa } \\
\text { y dice el sujeto a } \\
\text { partir de las formas } \\
\text { de expresión y } \\
\text { contenido. Es } \\
\text { la apropiación } \\
\text { y creación de } \\
\text { significados que } \\
\text { solo puede hacer el } \\
\text { sujeto si conoce las } \\
\text { formas. }\end{array}$ \\
\hline
\end{tabular}

Fuente. Elaboración propia con base en Narváez (2013) 
Según estos presupuestos teóricos, Narváez (2013) propone que la cultura, como código, puede dividirse entre cultura popular y cultura alfabética. Por un lado, la cultura popular corresponde a la tradición oral- icónica y narrativa asociada al mito, y por el otro, la cultura alfabética corresponde a la tradición escrita, argumentativa y abstracta propia de la tradición ilustrada.

\begin{tabular}{|l|l|}
\hline \multicolumn{1}{|c|}{ Cultura popular } & \multicolumn{1}{c|}{ Cultura alfabética } \\
\hline Oral - icónica - narrativa & Alfabética - argumentativa \\
\hline
\end{tabular}

Fuente. Elaboración propia con base en Narváez (2013)

La cultura popular se apropia en la interacción cotidiana, pues al nacer los seres humanos llegan a un ambiente cultural hecho, éste se apropia y se recrea por exposición en un espacio físico concreto, y por el interés explícito de los miembros de la comunidad para que los recién llegados apropien los significados compartidos. En consecuencia, las formas de expresión y contenido privilegiadas son la oralidad, la imagen y la narrativa que conforman el código de dominio mayoritario, es decir, un código común. En cambio, la cultura alfabética es un código elaborado que no se aprende por exposición en la vida cotidiana, sino a través del estudio.

Lo que Narváez (2013) llama cultura alfabética corresponde a la tradición del pensamiento que ha dado origen al desarrollo de la ciencia y la tecnología que caracteriza la modernidad. En síntesis, se trata de la cultura académica que describe Mockus y sus colegas (1995) en las fronteras de la escuela, "la cual se caracteriza por un modo de conocer que se codifica por escrito, se basa en la discusión racional científica, el cálculo, el diseño y la acción orientada y organizada racionalmente" (p. 63). Esta cultura debe ser estudiada a través de métodos que la escuela ha desarrollado en su devenir histórico. De manera que, para promover, apropiarse y recrear la cultura alfabética, ha sido necesario construir un ambiente escolar que ha hecho posible apropiar un código minoritario y restringido pero muy valorado como forma privilegiada de conocer.

Narváez (2012) define la cultura alfabética como:

Es una cultura gramaticalizada que exige el conocimiento de las reglas antes que la interpretación y la producción de los textos. Es decir, no se aprende por imitación, por el ejemplo, ni por la representación icónica, sino por el dominio de las reglas combinatorias de las unidades discretas. Es una cultura que requiere ser enseñada, transferida del que sabe al que no sabe; es una cultura que necesita el estudio, antes que el aprendizaje. Esto es lo que le da ese carácter especializado y exige casi siempre la institucionalización. (p. 87)

Siguiendo esta perspectiva, el conocimiento erudito que refiere Comenio en la Didáctica Magna, corresponde en esta clasificación a la cultura alfabética, mientras que el conocimiento común, que hasta cierto punto podemos asociar a la tesis de Rousseau - en cuanto al uso que puede hacerse de los ambientes comunes para desarrollar una tarea educativa - corresponde a la cultura popular. Así, el código de la cultura alfabética y la cultura popular se convierte en un factor diferenciador de 
los ambientes de aprendizaje, que sirve tanto a la caracterización de los ambientes históricamente constituidos, como al diseño de nuevos ambientes a la medida de las necesidades de los estudiantes.

\section{Clasificación culturalista, moderada, de los ambientes de aprendizaje}

Hasta este punto se ha expuesto la diferencia entre naturaleza y ambiente, la relación entre el hombre, el lenguaje y la cultura, y el ambiente humano como construcción estética sensorial, aspectos que permiten comprender el concepto de ambiente de aprendizaje. Con ello, resulta posible sugerir una clasificación que arroje luces sobre las posibilidades y condiciones para el diseño de ambientes de aprendizaje, en consideración del ambiente escolar moderno y la perspectiva culturalista anunciada en la parte inicial del texto. Esta clasificación responde al propósito de integrar procesos de educación formal y no formal, con base en la codificación preponderante de los ambientes de aprendizaje, y a la integración de las TIC como sustancia expresiva que soporta el acceso e intercambio de información en cualquier ambiente de aprendizaje; a esta última perspectiva, se suma la particularidad de los procesos de automatización que se puede lograr con algoritmos para simular un ambiente de aprendizaje. En orden de exposición, puede hablarse, entonces, de los ambientes naturales, los ambientes cotidianos-populares y alfabéticos, el ambiente alfabético pedagógico y los ambientes simulados con multimedia.

\subsection{Ambientes naturales}

Aunque el ser humano modifica la naturaleza para su beneficio, no siempre puede controlar y definir todos los fenómenos, por tanto, hay aspectos que escapan a su control que provocan procesos de adaptación cultural. Por ejemplo, las variables del clima, la vegetación, la topografía, la relación con otras especies, estos aspectos generan respuestas adaptativas que se inscriben en la condición de organismo vivo de los seres humanos.

\subsection{Ambientes cotidianos - populares}

También puede apelarse a las condiciones del ambiente, estos se constituyen como acumulados de la cultura en la vida cotidiana, tales como: la arquitectura, la producción y consumo de alimentos, el vestuario, las formas de consumo cultural, entre otros, que exigen una respuesta interpretativa de los sujetos. De hecho, la apropiación de todos los elementos del ambiente constituye el primer aspecto de la educación como condición antropológica cultural. Porque, tal como lo asegura Kant (2003), de esta forma los seres humanos dejan de ser unos miembros de la especie para convertirse en sujetos de la cultura. En términos comunicativos, los ambientes cotidianos - populares están codificados de forma sintética, oral, icónica y narrativa. 


\subsection{Ambientes alfabéticos no escolares}

Los ambientes alfabéticos no escolares son aquellos constituidos históricamente con el uso de la tradición alfabética e ilustrada con fines de información. Estos ambientes están configurados para estructurar el pensamiento analítico, categorial y abstracto que caracteriza la episteme moderna. Piénsese en la arquitectura y en la forma de relacionarse en ambientes como las bibliotecas, los museos, las universidades, los colegios, las escuelas, entre otros. En estos ambientes, hay una especial relación con el saber que lo hace diferente a los ambientes de la cultura popular. Por ejemplo, en la biblioteca se alojan los libros que condensan la cultura ilustrada y a la vez se establecen códigos de interacción que intentan favorecer su apropiación; por ejemplo, no alzar la voz y aislarse para estudiar, entre otros.

Por su parte, en los museos se exponen piezas de historia y de arte de acuerdo con una clasificación (curaduría) dirigida por criterios especializados, porque de ninguna manera las piezas están expuestas por azar; siempre hay una lógica y un propósito ligado al pensamiento categorial e ilustrado, así se quiera mostrar de otra forma. Así como en la biblioteca, en el museo también hay una distribución del espacio y el tiempo, pero para la contemplación. La forma en que se recorren los pasillos y se disponen uno a uno los cuadros, las esculturas, los artefactos, entre otras piezas de exposición, configuran una intencionalidad que de hecho tiende a volverse concreta con textos de carácter informativo y explicativo. De esta forma, los ambientes alfabéticos aparecen codificados preponderantemente de forma abstracta y alfabética; aunque, también de forma oral, icónica, narrativa, siempre y cuando expresen el tipo de pensamiento analítico - categorial que identifica la tradición ilustrada.

\subsection{Ambiente alfabético - pedagógico}

Por su parte, el ambiente alfabético - pedagógico corresponde al "taller de humanidad" descrito por Comenio para enseñar todo a todos. Se trata del ambiente que configura una forma particular de relacionarse, con el fin de apropiarse y recrear la tradición ilustrada, que conlleva una forma de pensar y comportarse; la forma de pensar y comportarse propia del proyecto moderno. El ambiente alfabético - pedagógico se orienta por los mismos elementos del ambiente cotidiano - alfabético, pero agrega una serie de ejercicios que buscan favorecer la autorregulación, la participación en lo público, el interés por el bien común (de acuerdo con el proyecto liberal de la educación) y, preponderantemente, el aprendizaje de los conocimientos desarrollados por la tradición científica moderna. Este ambiente se suele configurar con base en una arquitectura, una distribución del espacio y unas formas de relación que se asocian a una manera de relacionarse consigo mismo y los demás. Se diferencia del ambiente cotidiano - alfabético en su forma privilegiada de codificación, ya que propende por la apropiación y la recreación de la cultura alfabética; especialmente, la cultura codificada en textos de contenido abstracto y argumentativo. El dominio de este código permite continuar 
apropiando y recreando esta cultura en otros ambientes de aprendizaje. Por tanto, parece ser la condición sine qua non para que el diseño de nuevos ambientes de aprendizaje con el uso de las TIC y la integración de los ambientes no formales termine favoreciendo el aprendizaje de las materias STEAM.

\subsection{Ambientes simulados con multimedia}

Por último, se presenta los ambientes simulados con multimedia con fines recreativos, artísticos y pedagógicos. Se trata de una representación de condiciones ambientales que conllevan ciertos contenidos culturales pero que no tienen correlato perceptivo similar; por ejemplo, la escenografía de una obra de teatro, la "ambientación" del mar en un aula de clase, la reproducción electrónica de la cultura en equipos de cómputo, entre otros. En este sentido, la idea de diseño de ambientes simulados con multimedia pasa por la intencionalidad manifiesta de generar el reconocimiento de variables asociadas al proceso de apropiación cultural que experimenta los sujetos vinculados a ellos. En este nivel se podría incluir el llamado: diseño de ambientes virtuales de aprendizaje que se apoya en las TIC.

Aunque los ambientes simulados con multimedia pueden incluir las formas y el contenido de la cultura popular o alfabética, siempre estarán limitados a la estimulación de los sentidos de la visión y la audición ${ }^{5}$, porque con la figuración y simulación de estos ambientes aún no es posible reproducir la experiencia química del olfato, el gusto y la experiencia táctil ligada a la caricia y las señales de cercanía con el otro, de la cual depende, en gran medida, la integración de la experiencia del tiempo, el ritmo y el espacio en los seres humanos.

Asimismo, es preciso decir que el diseño de ambientes simulados con multimedia no constituye de ninguna manera un "espacio virtual"; porque, aunque esta denominación sugiere una inmaterialidad, en realidad se trata de un conjunto de retenciones terciarias soportadas en un dispositivo de almacenamiento electrónico que se reproducen por la acción humana en interfaces. En palabras de Stiegler (2001):

Aquí se llama "espacios virtuales" a los conjuntos retencionales de datos, conservados físicamente en soportes digitales inaccesibles sin la mediación de un dispositivo de representación de estas informaciones y cuya imagen intuitiva se construye para representar y hacer manipulables, por medio de interfaces, estos estados de materia ilegibles para una conciencia no equipada - en ningún caso se trata de "inmaterialidad"-. Se trata de otra materialidad. (p.226)

Con la descripción de los ambientes simulados con multimedia se completa la clasificación culturalista sugerida y, a la vez, se ofrecen unas pistas para el diseño de ambientes de aprendizaje a cargo del maestro. Para sintetizarlas en un párrafo, es posible decir lo siguiente:

5 Aunque algunos sistemas incluyen un efecto de vibración vinculada a la experiencia táctil. 
En primer lugar, es fundamental definir la intencionalidad educativa del ambiente que se quiere diseñar porque esto determinará las condiciones de posibilidad de la integración entre espacios formales y no formales. Si la intención es observar, analizar y apropiar la cultura popular, la integración de los ambientes cotidianos puede resultar una opción muy valiosa. En segundo lugar, si la intencionalidad es reconocer expresiones de la tradición ilustrada, puede resultar conveniente la integración de los ambientes alfabéticos no escolares. En ambos casos, la intencionalidad estará mediada por la codificación, ejercicios y técnicas del ambiente alfabético pedagógico, en cuanto esté concentra gran parte de la intencionalidad educativa formal.

\section{El debilitamiento de las fronteras de la escuela y el fracaso del aprendizaje de los "saberes útiles"}

Con la exposición realizada hasta ahora, se concluye que, para el diseño de nuevos ambientes de aprendizaje, es preciso considerar la codificación que enmarca la construcción estética sensorial, los elementos físicos, la temporalidad y el modo de interacción de los sujetos en un territorio delimitado, física e históricamente.

Así mismo, es posible concluir que el diseño de nuevos ambientes de aprendizaje puede producir una apropiación y recreación de los contenidos disciplinares, codificados de forma alfabética y argumentativa como las materias steam, si la experiencia escolar moderna favorece primero una forma particular de autorregulación y relación con los demás.

El ambiente escolar moderno está constituido por condiciones de tiempo, espacio e interacción que conllevan la construcción y reconstrucción de una codificación de conocimientos útiles para el mercado. Si se reconfigura, también se altera la codificación que acarrea el modo de interacción entre los sujetos que conduce a la apropiación del código de la cultura alfabética. En otros ambientes, también se apropian y recrean aspectos de la cultura muy valiosos y necesarios para el propósito educativo, pero, sobre todo, en el ambiente escolar moderno se producen condiciones específicas para el aprendizaje del código alfabético argumentativo que sirven para el desarrollo de opciones productivas y competitivas, pero crean el hábito para apropiarse de los saberes escolares en otros ambientes de aprendizaje.

\section{Referencias}

Chomsky, N. (1988). Language and problems of knowledge. The Managua lectures, (ed. cast.: El lenguaje y los problemas del conocimiento. Conferencias de Managua I, Visor, Madrid

Comenio, J. A. (1994). Didáctica Magna. $5^{a}$ ed. México: Editorial Porrúa.

Commission European (2008). "The Use of ICT to Support Innovation and Lifelong Learning for All - A Report on Progress". En European Commission. Recuperado de: http://www.europarl. 
europa.eu/registre/docs_autres_institutions/commission_europeenne/sec/2008/2629/ COM_SEC(2008)2629_

De Saussure, F. (1982). Curso de lingüistica general. México: Nuevo mar,

Depaepe, M., y Simon, F. (2008). Sobre la pedagogización... Desde la perspectiva de la historia de la educación. Espacios en Blanco. Revista de Educación, (18), pp. 101-130.

Drucker, P. (1969). The age of discontinuity. Guidelines to our changing society. New York: Harper \& Row.

Duarte, J. (2003). Ambientes de aprendizaje: una aproximación conceptual. Estudios Pedagógicos, núm. 29, 2003, pp. 97-113 Universidad Austral de Chile Valdivia, Chile.

Eco, U. (2000). Tratado de semiótica general. (5 Ed) España: Lumen, SA

Foucault. M. (1978). Las palabras y las cosas. Madrid: Siglo XXI

García-chato, G. (2014). Ambiente de aprendizaje: su significado en Educación Preescolar. En revista de Educación y desarrollo, (29). Abril-junio.

Geertz, C. (1973). Descripción densa: hacia una teoría interpretativa de la cultura. Comunicación II - Cátedra Martini

Grupo Banco Mundial. (2016). “Dividendos Digitales. Panorama General”. Informe sobre el desarrollo mundial. Washington DC: Banco Internacional de Reconstrucción y Fomento /Banco Mundial. Recuperado de: http://documents.worldbank.org/curated/ en/658821468186546535/pdf/102724-WDR-WDR2016Overview-SPANISHWebResBox-394840B-OUO-9.pdf

Hjelmslev, L. (1974) Prolegómenos a una teoría del lenguaje. España, Madrid: Gredos.

Instituto Nacional de Tecnologías Educativas y de formación del profesorado (INTEF). (2017). Resumen informe horizon. Recuperado de: http://educalab.es/ documents/10180/38496/Resumen_Informe_Horizon_2017/44457ade-3316-418e-9ff9fd5e86fc6707

Leroi-Gourhan, A. (1971). El gesto y la palabra. (Traducción Felipe Carrera). Caracas: Ediciones de la Biblioteca Universidad Central de Venezuela

Lévi-Strauss, C. (1993). Las estructuras elementales del parentesco. Vol. I. Barcelona: Planeta Kant, I. (2003) Pedagogía. Madrid: Akal.

Marcondes, M. (2004). A educação não-formal e a relação escola-comunidade. EccoS Revista Científica, 6 (2), 39-65.

Marín-Díaz, D., y Noguera, C. (2017). En defensa de la experiencia escolar. Fortalecer las fronteras de la escuela. ETD - Educação Temática Digital. (19). 607. 10.20396/etd. v19i4.8648826. 
Noguera, C. y Marín-Díaz, D. (2017). Saberes, normas y sujetos: cuestiones sobre la práctica pedagógica. Educar em Revista, (66), 37-56. Recuperado de https://dx.doi. org/10.1590/0104-4060.53866

Mominó, Josep y SIGALÉS, Carles (coords) (2016). El impacto de las TIC en la educación. Barcelona. España. UOC Editorial.

Mockus, A., Hernández, C., Granes, J., Charum, J., y Castro, M. (1994) Las fronteras de la escuela. En Revista Educación y Pedagogía Núm 12 y 13, pp. 368-381. Bogotá: Sociedad Colombiana de Pedagogía.

Narváez, A. (2012). Cultura alfabética, educación y desarrollo. Pedagogía Y Saberes, (36), 81.92. Recuperado de https://doi.org/10.17227/01212494.36pys81.92

Narváez, A. (2013). Educación y comunicación: del capitalismo informacional al capitalismo cultural. $1^{a}$ ed. Bogotá: Universidad Pedagógica Nacional, Doctorado Interinstitucional de Educación (DIE).

Raichvarg, Daniel (1994): "La educación relativa al ambiente: Algunas dificultades para la puesta en marcha”. En: Memorias Seminario Internacional. La Dimensión Ambiental y la Escuela. Santafé de Bogotá, Serie Documentos Especiales MEN.

Reboratti, C. (2000). Ambiente y sociedad. Conceptos y relaciones. Buenos Aires: Ariel

Rousseau, J. J. (1984). Emilio o de la educación. México: Porrúa.

Salinas, J. (2008). Nuevos ambientes de aprendizaje para una sociedad de información. Universitat de les illes balears. Recuperado de https://www.ses.unam.mx/curso2008/pdf/ Salinas.pdf

Sierra Pérez, J. (2005). Aprendizaje autónomo: eje articulador de la educación virtual. Revista Virtual Universidad Católica del Norte, (14)

Simons, M., y Masschelein, J. (2014). Defensa de la escuela. Una cuestión pública. Buenos Aires: Miño y Dávila

Stiegler, B. (2001). La técnica y el tiempo. Tomo 3: El tiempo del cine y la cuestión del malestar. Guizpukoa. Cultura Libre.

Tröhler, D. (2014). Pestalozzi y la educacionalización del mundo. Barcelona, Octaedro.

Vélaz de Medrano, C (2009) Nuevos ambientes de aprendizaje para el desarrollo profesional docente. En: Veláz de Medrano, C. y Vaillant, D. (ed.). Aprendizaje y desarrollo profesional docente. Madrid: OEI y Fundación Santillana. Recuperado de http://www.oei.es/metas2021/APRENDYDESARRPROFESIONAL.pdf

Virno, P. (2005). Cuando el verbo se hace carne: Lenguaje y naturaleza humana, trad. Eduardo Sadier, Madrid: Traficantes de sueños 